\title{
Development of an electrochemical maltose biosensor
}

\section{Carlos Morón ${ }^{1, a}$, Enrique Tremps ${ }^{1, b}$, Alfonso Garcia ${ }^{1, c}$, Jose Andrés Somolinos ${ }^{2, d}$}

\author{
${ }^{1}$ Dpto. Tecnología de la Edificación, E.U. Arquitectura Técnica, Av. Juan de Herrera \\ ${ }^{2}$ Dpto. Sistemas Oceánicos y Navales, ETSI Navales \\ Sensors and Actuators Group, Universidad Politécnica de Madrid, Spain \\ acarlos.moron@upm.es, benrique.tremps@upm.es, calfonso.garciag@upm.es, \\ joseandres.somolinos@upm.es,
}

Keywords: maltose biosensor, Faradic current.

\begin{abstract}
In this work, electrochemical maltose biosensors based on mutants of the maltose binding protein (MBP) are developed. A ruthenium ${ }^{\mathrm{II}}$ complex $\left(\mathrm{Ru}^{\mathrm{II}}\right)$, which is covalently attached to MBP, serves as an electrochemical reporter of MBP conformational changes. Biosensors were made through direct attachment of $\mathrm{Ru}^{\mathrm{II}}$ complex modified MBP to gold electrode surfaces. The responses of some individual mutants were evaluated using square wave voltammetry. A maltose-dependent change in Faradic current and capacitance was observed. It is therefore demonstrated that biosensors using generically this family of bacterial periplasmic binding proteins (bPBP) can be made lending themselves to facile biorecognition element preparation and low cost electrochemical transduction.
\end{abstract}

\section{Introduction}

The development of most biosensors involves the identification of naturally occurring macromolecules that provide the desired analyte specificity (typically an enzyme or antibody) [1]. Once identified, a suitable signal transduction and detection methodology needs to be adapted to the macromolecule in question [2]. This is especially true for affinity biosensors, since catalytic biosensors usually are based on the detection of an ubiquitous reaction product. Although cumbersome, effective affinity biosensors have been developed in this way but each device is unique and requires substantial development time since transduction of conformational changes is not straightforward and needs to be adapted to the biorecognition molecule. To overcome this limitation, new approaches are being developed in which protein engineering is used to adapt the signal-transduction properties of biological molecules to the detector instrumentation and the transduction chemistry of the configuration, rather than adapting instruments and chemistry to the unique requirements of each natural molecule [3]. This is achieved by integrating functional groups that provide a simple signal-transduction mechanism between the detector and the protein. Protein engineering has been used to construct fluorescent [4] and electrochemical [5] sensors based on the maltose binding protein that is known to undergo significant conformational changes upon maltose binding. The aim of this work is to further examine the adaptation of fluorescent semiconducting nanoparticle-based biosensors for maltose to electrochemically transduced maltose biosensors and to develop a reagentless electrochemical biosensor using maltose-binding protein (MBP) directly adsorbed to bare gold electrodes and square wave voltammetry (SWV) as a detection method.

\section{Experimental method}

To achieve our objective the MBP-MT plasmids were transformed into BL21-DE3 E. coli competent cells. Four surface cysteine mutant MBP-MTs were generated and attachment of the $\mathrm{Ru}^{\mathrm{II}}$ complex to mutant MBP-MTs was performed. [Ru ${ }^{\mathrm{II}}(1,10$-phenanthroline-5-maleimide) $\left.\left(\mathrm{NH}_{3}\right)_{4}\right]\left[\left(\mathrm{PF}_{6}\right)\right]_{2}$ (1.0 equivalents, $10 \mathrm{mM}$ stock in acetonitrile was added to the $\mathrm{Cd}_{2}{ }^{+}$ion ${ }^{-}$protected protein solution and reacted for two hours. The ruthenated protein sample was quenched with 100 
$\mu \mathrm{M}$ 2-mercaptoethanol and purified by gel filtration chromatography. $\mathrm{The} \mathrm{Cd}_{2}{ }^{+}$ions were removed by treatment with $0.1 \mathrm{mM}$ EDTA and $0.1 \mathrm{mM} \mathrm{1,10-phenanthroline} \mathrm{at} 4{ }^{\circ} \mathrm{C}$ for 2-24 hours, followed by purification with gel filtration chromatography. The resulting proteins were analyzed by absorbance spectrophotometry monitoring the ratio of the $\mathrm{Ru}{ }^{\mathrm{II}}$ complex $490 \mathrm{~nm}$ and the protein 280 $\mathrm{nm}$. Only one $\mathrm{Ru}^{\mathrm{II}}$ complex can be linked to the protein surface. The protein labeled percentage can be estimated using the absorbance values of both, protein and $\mathrm{Ru}^{\mathrm{II}}$ complex (1:1 ratio).

Gold ball electrodes were produced by the method of Creager [6]. Gold wire $(0.25 \mathrm{~mm}$ diameter) was threaded through a pulled glass capillary and flame annealed. The gold ball-capillary junction was sealed with non-conducting epoxy. The gold ball electrode was immersed in $2 \mathrm{~mL}$ of supporting electrolyte solution $(0.2 \mathrm{M}$ sodium phosphate, $\mathrm{pH} 7.5,100 \mathrm{mM} \mathrm{KCl})$. After the addition of $0.5 \mathrm{~mL}$ protein solution $(10 \mu \mathrm{M})$, incubation proceeded under stirring, for 20 minutes. During this process, the metallothionine domain (MT) attached the $\mathrm{Ru}^{\mathrm{II}}$-MBP-MT to the gold surface.

Electrochemical experiments using a three-electrode one-compartment cell were conducted using a potentiostat ( $\mathrm{CH}$ Instruments, Model 812A). The electrochemical measurements were conducted using an $\mathrm{Ag} / \mathrm{AgCl}$ reference electrode and a platinumcounter electrode (Cypress systems: 66-EE008 and 66-EE011, respectively). The potential range of cyclic voltammetry was between 0.0 and $0.6 \mathrm{~V}$ (vs. $\mathrm{Ag} / \mathrm{AgCl}$ ) with a scan rate of $50 \mathrm{mV} / \mathrm{s}$. Square wave voltammograms (SWV) were registered in the potential interval $0.0-0.6 \mathrm{~V}$ (vs. $\mathrm{Ag} / \mathrm{AgCl})$, under the following conditions: potential increment, $1 \mathrm{mV}$; potential amplitude, $10 \mathrm{mV}$; pulse frequency, $15 \mathrm{~Hz}$ which was optimized in relation with the peak definition. SWV currents of $\mathrm{Ru}^{\mathrm{II}}$ complex modified MBP-MTs were subtracted from unmodified MBP-MT SWVs and linear interpolations between +100 and $+550 \mathrm{mV}$ vs. $\mathrm{Ag} / \mathrm{AgCl}$ were used to enhance Faradic current in order to calculate the correlated current.
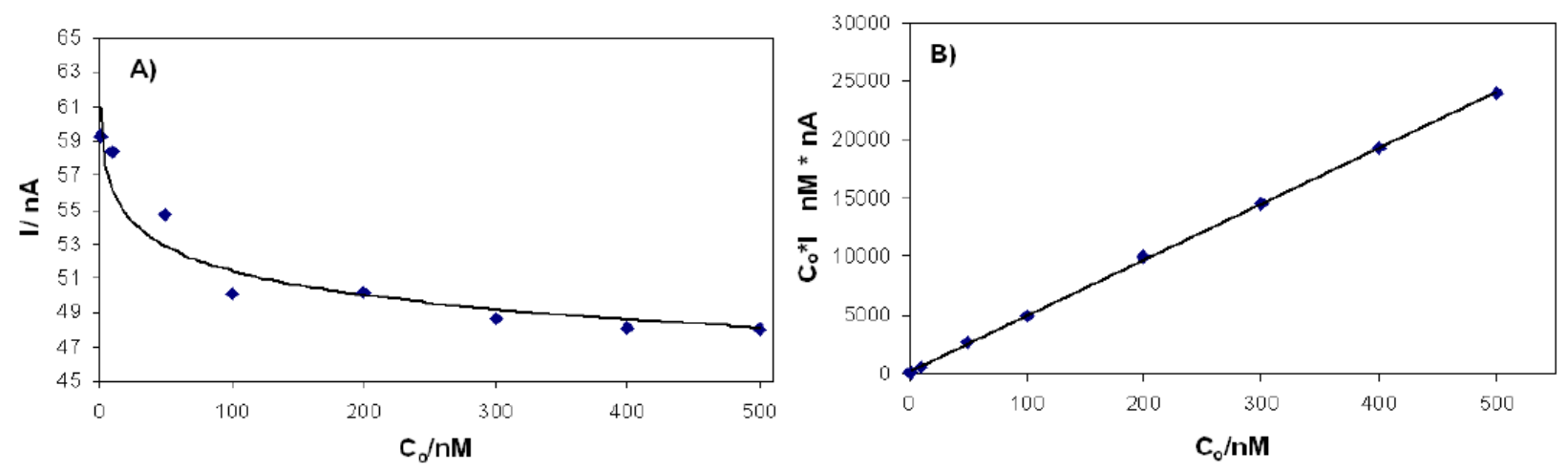

Fig. 1. Maltose titration: A) Amperometric titration data using SWV was able to detect small variations of maltose concentration. The corresponding titration curve has shown progressive decrease of current as a function of maltose concentrations. B) Maltose-dependent change current, which was fit to the Langmuir isotherm to derive $K D=44 \mathrm{nM}$.

\section{Results and discussion}

The cyclic voltammogram of gold electrode in $0.1 \mathrm{mM}$ [RuII(1,10-phenanthroline-5maleimide) $\left.\left(\mathrm{NH}_{3}\right)_{4}\right]\left[\left(\mathrm{PF}_{6}\right)\right]_{2}$ shows anodic and cathodic peaks located at 383 and $296 \mathrm{mV}$ (vs. $\mathrm{Ag} / \mathrm{AgCl}$ ), respectively. The formal potential $\left(\mathrm{E}^{\circ}\right)$ is $340 \mathrm{mV}$ (vs. $\mathrm{Ag} / \mathrm{AgCl}$ ) which is in agreement with the literature value for this complex [7]. The peak separation of $120 \mathrm{mV}$ is indicative of a quasi-reversible one-electron heterogeneous electron transfer process. Cyclic voltammetry experiments were performed on the Ru-MBP-MT system. Only a weak Faradic response was observed suggesting minimal Ru-MBP-MT loading on the gold electrode surface. Square wave voltammetry did show a significant Faradic response and was used to characterize the Ru-MBP-MT system in this report. Some different mutations of maltose binding protein were evaluated in this work. Each mutation has shown different electrochemical response using SWV. This technique has 
been found to be useful for the electrochemical analyses of diffusionless systems and, in this report, for studies of conformational changes of a protein adsorbed to a solid surface. When the protein is adsorbed, the current response of the Faradic electrochemical signal should depend on the distance of the electrochemical label from the surface, while the peak potential should be directly correlated to the electrochemical label reduction potential.

The individual mutations were used to modify gold electrodes as described in the experimental section. SWV experiments were performed with two types of modified electrodes: MBP-MT with (sample) and without (control) the $\mathrm{Ru}^{\mathrm{II}}$ complex. For each type of electrode, SWV was recorded before and after the addition of $10 \mu \mathrm{M}$ maltose, which was determined previously to be a saturating concentration.

Maltose titration studies were also performed. Amperometric titration data using SWV showed discernible response even to small variations of maltose concentration. The corresponding titration curve has shown progressive decrease of current as a function of maltose concentrations, which is in accordance with the literature. The binding affinity based interactions between maltose and MBPMT will be evaluated. In figure 1A the peak current, $I$, is plotted as a function of the bulk concentration co. Under this conditions the dissociation constant $(K D=44 \mathrm{nM})$ was obtained from the curve fitting (Figure 1B) using the Langmuir isotherm.

$$
\theta=K A c 0 /(1+K A c o)
$$

Where, $\theta$ is the fractional coverage defined as $\Gamma / \Gamma \mathrm{m}$ ( $\Gamma \mathrm{m}$ is the Ru-MBP-MT maximum coverage), $c o$ is the bulk maltose concentration, and $K A$ is the affinity constant. The coverage $\Gamma$ of binding maltose is inversely proportional to the current $I$. In this case eq. 1 can be expressed as:

$$
I=\operatorname{Im}(K D+c o) / c 0
$$

Where $\mathrm{Im}$ is the maximum current of Ru-MBP-MT on bare gold electrode and $K D$ is the dissociation constant. $K D=1 / K A$.

The selectivity of the sensor was studied by SWV using as baseline, for this experiment, the signal recorded after the immobilization of MBP-MT-Ru on gold surface electrode. After the addition of different sugars (lactose, glucose and maltose respectively) a SWV was obtained (Figure $2)$. The MBP biosensor showed better selectivity for maltose $(66.8 \%)$ when compared to other substrates (insignificant signal for lactose and $7.1 \%$ for glucose). This finding confirmed the substrate selectivity of MBP-based electrochemical biosensor.

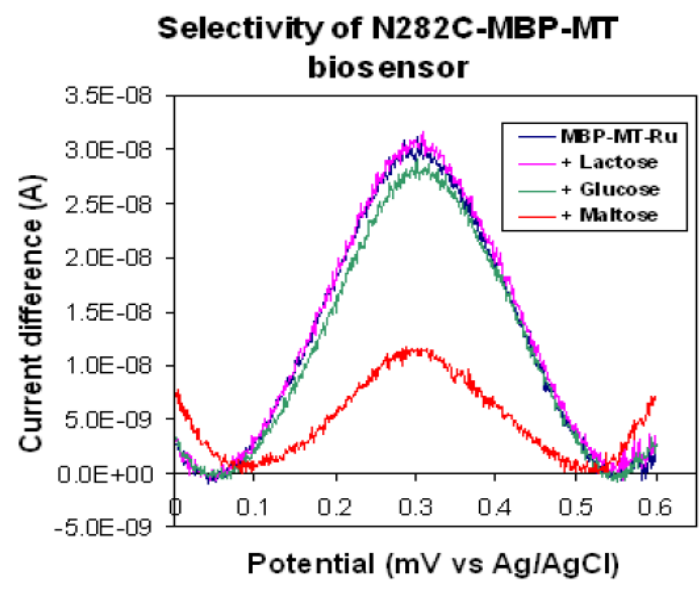

Selectivity of N282C-MBP-MT biosensor

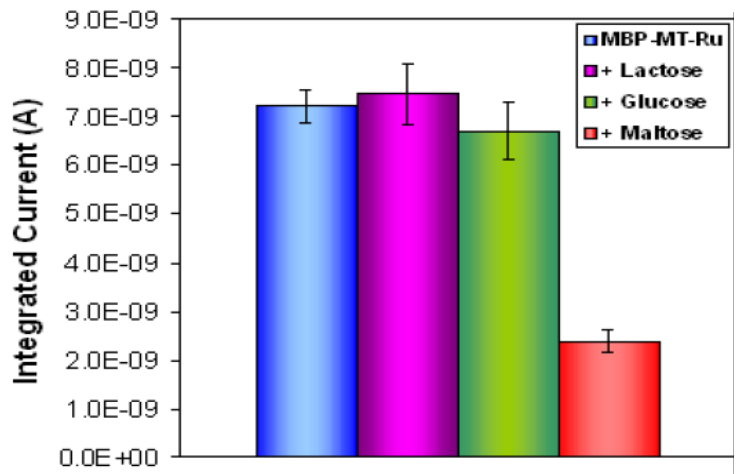

Figure 2. The selectivity of the sensor was obtained by SWV. The baseline for this experiment was the signal recorded after the immobilization of MBP-MT-Ru (blue) on gold surface electrode. After the addition of each sugar (lactose; pink, glucose; green and maltose; red respectively) the signal decrease was analyzed. The selectivity for maltose is $66.8 \%$ in comparison with other substrates (insignificant signal for lactose and $7.1 \%$ for glucose).

These findings are significant, in that a maltose-dependent electrochemical response with square wave voltammetry was observed. Square wave voltammetry requires less expensive electronics than $A C$ voltammetry. This analytical advance stems from, purposely, improved electron transport 
between the $\mathrm{Ru}^{\mathrm{II}}$ complex and the gold electrode provided by the metallothionin domain attachment chemistry.

\section{Conclusions}

Different $\mathrm{Ru}^{\mathrm{II}}$ complex attachment sites on the MBP-MT surface reported in the present work were surveyed using SWV. Each position has shown a different electrochemical signal according to the distance dependence effect, wherein, the conformational change was involved after the biorecognition process. N282C-MBP-MT showed a significant maltose-dependent signal (16.3 nA) that had a combined capacitive and Faradic contribution. Therefore, Ru-N282C-MBP-MT represents the best system for maltose biosensing with the electrochemical detection system employed. In addition, the direct adsorption of $\mathrm{Ru}^{\mathrm{II}}$ modified MBP-MT on bare gold electrodes was tested with satisfactory results. This suggests that the coupling between the modified proteins and gold electrode surface is a successful method for electrochemical detection. The previous results suggest that $\mathrm{N} 282 \mathrm{C}$ mutant has shown the best electrochemical signal, due to its reporter group location and the dynamic interaction between the reporter and the gold electrode surface.

The conformational changes involved in MPB-MT have been evaluated by SWV technique. The results suggest that SWV could be an attractive alternative for biosensing compared to the more complicated and expensive techniques such as ac voltammetry [5]. The apparent disassociation constant $(K D)$ reported by these studies, have shown a significant value of $44 \mathrm{nM}$, which represents an important improvement in affinity (10-15 fold increase) in comparison with previous electrochemical [5] and fluorescence [8] MBP biosensors. Previously, the surface of the gold electrodes used to be covered by a monolayer (linker), which increased the capacitance effect. In contrast, in our approach, the direct absorption of MBP-MT over the surface electrode reduces the capacitance effect and the Faradic current can be recorded without interferences.

The maltose selectivity and the non-specific interaction for two additional targets have been studied. The signal at saturated condition of maltose has shown a significant signal of $68 \%$ of the total maximum signal. The evaluation of non-specific targets has revelled a $7 \%$ of the total signal for glucose and an insignificant signal for lactose. The non-specific signal for glucose could be explained by the fact that glucose is the monomer of maltose, thus some partial signal could be expected.

In summary, this work provides a high affinity maltose-dependent biosensor for studying the allosteric mechanism of maltose binding protein adsorbed to gold surfaces using electrochemical techniques. The results suggest that conformational changes represent a successful intrinsic transduction mechanism for biomolecules. In addition, this type of intrinsic transduction could be applied to the design and development of generic biosensors.

\section{References}

[1] A.P. Turner, I. Karube, G.S. Wilson. Biosensors: Fundamentals and Applications. 1987.

[2] Gopel, Sensors: A Comprehensive Survey. 1994.

[3] H.W. Hellinga, J.S.Marvin. Trends Biotech. 1998, 16, 183-189.

[4] J.S Marvin, E. E. Corcoran. PNAS. 1997, 94 4366-4371.

[5] D.E. Benson, D. W. Conrad, H.W. Hellinga. Science. 2001, 293, 1641-1644.

[6] S.E. Creager, T.T. Wooster. Anal. Chem. 1998, 70, 4257-4263.

[7] S.A. Trammell, H.M. Goldston. Bioconj. Chem. 2001, 12, 643-647.

[8] M.G. Sandros, V. Shete, D.E. Benson. The Analyst, 2006, 131, 229. 